\title{
FRACTAL ANALYSIS OF TRABECULAR BONE: A STANDARDISED METHODOLOGY
}

\author{
IAN PARKINSON AND NICK FAZZALARI
}

Division of Tissue Pathology, Institute of Medical and Veterinary Science, Adelaide, South Australia, Department of Pathology, University of Adelaide, South Australia (Accepted December 16, 1999)

\begin{abstract}
A standardised methodology for the fractal analysis of histological sections of trabecular bone has been established. A modified box counting method has been developed for use on a PC based image analyser (Quantimet 500MC, Leica Cambridge). The effect of image analyser settings, magnification, image orientation and threshold levels, was determined. Also, the range of scale over which trabecular bone is effectively fractal was determined and a method formulated to objectively calculate more than one fractal dimension from the modified Richardson plot. The results show that magnification, image orientation and threshold settings have little effect on the estimate of fractal dimension. Trabecular bone has a lower limit below which it is not fractal $(\lambda<25 \mu \mathrm{m})$ and the upper limit is $4250 \mu \mathrm{m}$. There are three distinct fractal dimensions for trabecular bone (sectional fractals), with magnitudes greater than 1.0 and less than 2.0. It has been shown that trabecular bone is effectively fractal over a defined range of scale. Also, within this range, there is more than 1 fractal dimension, describing spatial structural entities. Fractal analysis is a model independent method for describing a complex multifaceted structure, which can be adapted for the study of other biological systems. This may be at the cell, tissue or organ level and compliments conventional histomorphometric and stereological techniques.
\end{abstract}

Keywords: box counting, fractal analysis, histological sections, image analyser, methodology, sectional fractals, trabecular bone.

\section{INTRODUCTION}

The use of the fractal dimension to describe the structure of trabecular bone has been reported by a number of workers (Majumdar et al., 1993; Weinstein and Majumdar 1994; Chung et al., 1994; Fazzalari and Parkinson 1996, 1997). These studies employ either direct imaging of histological sections or digitisation of radiographic images. This study aims to provide an objective and reproducible methodology for the fractal analysis of histological sections of trabecular bone.

Classic fractal analysis involves estimation of the perimeter of an object using rulers of different lengths. As the size of the measuring unit decreases the estimated perimeter increases. These data plotted as log of perimeter versus log of measuring unit are linearly co-dependent. This is known as the Richardson plot and the fractal dimension $=\mathrm{D}$, where 1-D is the slope of the regression line.

Mandelbrot (1977) in formulating the principles of fractal geometry illustrated that natural objects have a finite range over which they are approximate fractal curves (Vicsek (1998) uses the term effective fractals). Therefore, it is imperative when estimating the fractal dimension that the size of the lower and upper limits of the structure have been determined.

As in conventional histomorphometry, the effect of sub-optimal grey level threshold detection of the trabecular bone, the resolution of the imaging system and the angle of presentation of the specimen to the imaging system potentially affect the fractal analysis. It is necessary to quantify the effect of changes to these parameters in order to specify the optimal conditions by which fractal analysis can be reproducibly performed.

Often the Richardson plot from which the fractal dimension is estimated does not form a simple straight line for natural objects (Kaye, 1989). Visual examination of the Richardson plot may reveal 2 or more successive straight-line segments, which by regression analysis fit the data better than a single line, ie. there is more than one fractal dimension (Fazzalari and Parkinson, 1997, 1998). These successive fractal dimensions are termed 'sectional' fractal dimensions.

Previously the 'sectional' fractals have been selected by subjective assessment, which is prone to operator variability (Fazzalari and Parkinson, 1997). Ideally, an objective method should be formulated to determine the straight-line segments on the Richardson plot so that 
there is uniformity within and between studies. The computational power of modern personal computers allows fast analysis of data, enabling application of complex iterative algorithms and statistical models.

The aims of this study are to devise an objective and reproducible methodology for the fractal analysis of histological sections of trabecular bone. In order to achieve this, a highly automated and integrated approach using software developed for a Quantimet ${ }^{\circledR}$ 500MC image analyser (Leica Cambridge, UK) will be presented. The principles of the methodology are applicable to any facility with access to programmable PC based image analysers.

\section{METHODS}

An automated box counting method was used on a Quantimet 500MC image analyser to estimate the fractal dimension. The box sizes range from 1 to 181 pixels in side length. The number of boxes containing the outline of the binary image is proportional to the perimeter of the object, (Chung et al., 1994; Fazzalari and Parkinson, 1996) such that the log of box size versus the log of the number of boxes produces a modified Richardson plot. The fractal dimension $=\mathrm{D}$, where $-\mathrm{D}$ is the slope of the line on the modified Richardson plot, for a range of box sizes.

Histological sections of trabecular bone from the femoral head were impregnated with silver and counterstained with van Gieson's stain to give maximum contrast between the bone matrix and marrow spaces (Fig. 1).

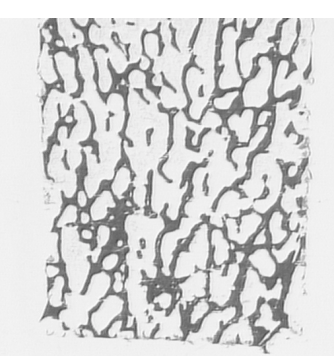

Fig. 1. Histological section of trabecular bone (1 pixel $=25 \mu \mathrm{m}$ ) when digitised on image analyser).

In order to determine the smallest box size, above which trabecular bone is effectively fractal, the fractal dimension was estimated for box sizes 1-4, 2-5, 3-6, .. 10-13 pixels, where box size of $1(5.4 \mu \mathrm{m})$ was the image analyser resolution. At this resolution, only individual trabeculae are analysed. The box sizes at which the fractal dimension was greater than 1.0 indicated the lowest image analyser resolution at which trabecular bone is an effective fractal.

The largest box size to be used in the estimation of the fractal dimension of trabecular bone was determined by calculating the standard error of the regression coefficient (R) from regression analysis of 1 to $4,5,6 \ldots 181$ pixels, where 1 pixel $=25 \mu \mathrm{m}$. The largest box size was determined to be the average box size closest to 181 pixels at which the standard error of $\mathrm{R}$ was at a minimum, for the histological sections.

The plateaux, which form when, for a range of box sizes the number of boxes counted is constant, were removed from the Richardson plot by 3 methods.

1. the first data point in each plateau was retained,

2. the last data point in each plateau was retained,

3. the data point that corresponded to the average box size in each plateau was retained.

The effect of image analyser settings and image orientation was determined. The optimal detection level was set using the 'flicker method' and then was set at \pm 1 to 5 grey levels. The object was rotated at $10^{\circ}$ intervals from 0 to $170^{\circ}$. The magnification was decreased to $80 \%, 60 \%, 40 \%$ and $20 \%$ of the initial resolution and the fractal dimension estimated.

Sectional fractal dimensions on the modified Richardson plot were determined using a split-line regression analysis. The assumption was made that there are 3 contiguous straight-lines, which accurately describe the data, on the modified Richardson plot. The data is analysed using an automated algorithm, which yields the 2 data points indicating the transition from one straight-line to the next. These are referred to as the 'break points' and are the same as the 'pivot points' described by Fazzalari and Parkinson (1997). The 'break point' estimates are then used to determine the 3 fractal dimensions (D1, D2 and D3).

\section{RESULTS}

The range of box sizes where the estimated fractal dimension becomes greater than 1.00 , is approximately $25 \mu \mathrm{m}$ (Table 1). Therefore, $25 \mu \mathrm{m}$ was selected as the lower limit at which trabecular bone is effectively fractal.

The mean box size for trabecular bone, where the standard error of $\mathrm{R}$ was a minimum, was 170 pixels or $4250 \mu \mathrm{m}$ (Fig. 2). This was selected as the largest box size. 


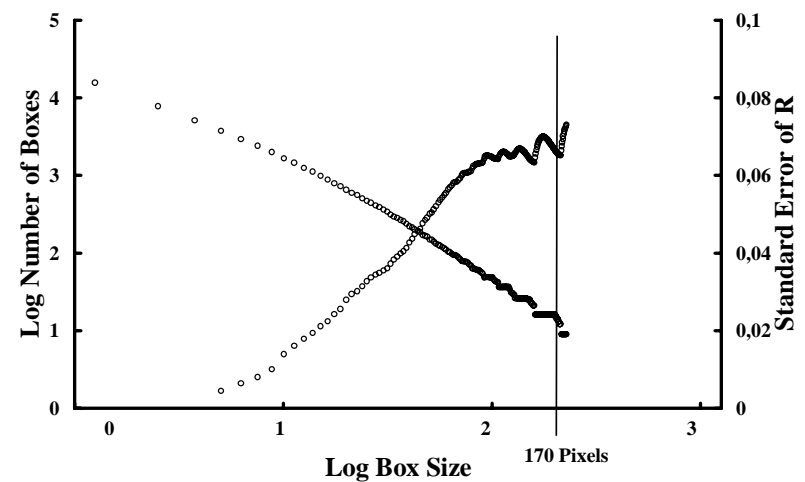

Fig. 2. Modified Richardson plot with standard error of $R$ overlaid.

Comparison of the three methods used to remove the plateaux show no statistical differences in the estimation of the fractal dimension (Table 2). The method, which selects the first point of each plateau, was adopted.

Table 2. Fractal Dimension (mean \pm sd) after replacement of each plateaux by retention of the first, the average and the last data points.

\begin{tabular}{c|ccc} 
& First & Average & Last \\
\hline Fractal & $1.55 \pm 0.05$ & $1.55 \pm 0.05$ & $1.54 \pm 0.05$ \\
Dimension & & &
\end{tabular}

The effect of change to image analyser settings, namely detection and magnification and the angle of presentation, show that for these parameters there was no significant change in the estimate of fractal dimension (Table 3, 4 and Fig. 3).

Table 1. Fractal dimension (mean \pm sd) of individual trabeculae for a range of box sizes.

\begin{tabular}{l|ccccc} 
Range $(\mu \mathrm{m})$ & $5.4-21.6$ & $10.8-27.0$ & $16.2-32.4$ & $21.6-37.6$ & $27.0-43.2$ \\
\hline Fractal Dimension & $1.00 \pm 0.01$ & $1.00 \pm 0.01$ & $1.01 \pm 0.02$ & $1.03 \pm 0.03^{*}$ & $1.05 \pm 0.04^{*}$ \\
Range $(\mu \mathrm{m})$ & $32.4-48.6$ & $37.8-54.0$ & $43.2-59.4$ & $48.6-64.8$ & $54.0-70.2$ \\
\hline Fractal Dimension & $1.05 \pm 0.05^{*}$ & $1.03 \pm 0.05^{*}$ & $1.06 \pm 0.07^{*}$ & $1.10 \pm 0.07^{*}$ & $1.12 \pm 0.09^{*}$ \\
${ }^{*}$ - denotes statistically significant difference to 1.00. & & &
\end{tabular}

Table 3. Fractal dimension (mean \pm sd) estimated at 1 to 5 grey-levels above and below the optimal detection level of trabecular bone.

\begin{tabular}{l|cccccc} 
Detection & Optimal & Up1 & Up2 & Up3 & Up4 & Up5 \\
\hline Fractal Dimension & $1.43 \pm 0.04$ & $1.45 \pm 0.03$ & $1.44 \pm 0.04$ & $1.43 \pm 0.04$ & $1.45 \pm 0.04$ & $1.45 \pm 0.04$ \\
Detection & & Down1 & Down2 & Down3 & Down4 & Down5 \\
\hline Fractal Dimension & & $1.43 \pm 0.03$ & $1.42 \pm 0.01$ & $1.41 \pm 0.02$ & $1.42 \pm 0.02$ & $1.41 \pm 0.02$
\end{tabular}

Table 4. Fractal dimension (mean \pm sd) estimated with changing initial magnification.

\begin{tabular}{c|ccccc} 
Magnification (\%) & 100 & 80 & 60 & 40 & 20 \\
\hline Fractal Dimension & $1.53 \pm 0.01$ & $1.54 \pm 0.01$ & $1.57 \pm 0.01$ & $1.55 \pm 0.05$ & $1.52 \pm 0.06$
\end{tabular}

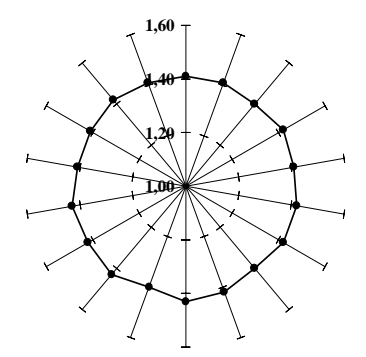

Fig. 3. Polar plot of fractal dimension estimated at different angles of presentation. 
There were 3 sectional fractals (D1, D2 and D3). The magnitudes were between 1.0 and 2.0 with $\mathrm{D} 1$ less than D2, which is less than D3 (Fig. 4).

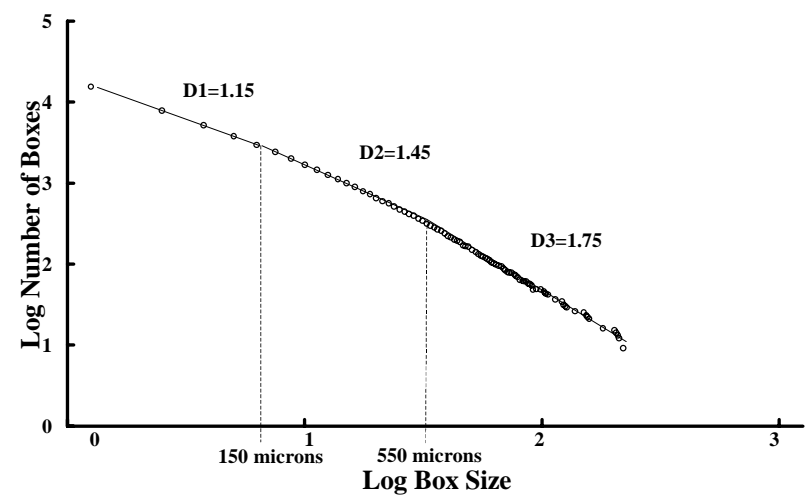

Fig. 4. Modified Richardson plot with 3 sectional fractal dimensions and 2 'break points'.

\section{DISCUSSION}

The results define a methodology for the standardised estimation of sectional fractal dimensions of histological sections of trabecular bone. In particular, the studies determine the lower and upper limits, within which trabecular bone is effectively fractal. The effect of changes to image analyser settings and specimen orientation on the estimation of fractal dimension was determined. Also, an objective method for determining the 'break points', which define sectional fractal dimensions, is established.

The use of individual trabeculae in the study that determined the lower limit, at which trabecular bone is effectively fractal, allowed a close examination of the surface texture of the trabeculae. This enabled the box size $(25 \mu \mathrm{m})$ for fractal analysis to be set at a value, which resolves the smallest features of interest. The largest box size $(4250 \mu \mathrm{m})$ used for fractal analysis was determined to be the value, above which variance in the data had increased above the minimum value and was the magnitude of the largest structure of interest.

The plateaux on the modified Richardson plot are present in all studies using a box counting method for fractal analysis. The loss of linearity in the data if they are not removed, significantly affects the estimation of the fractal dimension. Buzckowski et al. (1997) has described the mathematical convention, whereby the first point in a plateau is equivalent to the average of the values in the plateau and this method maintains the box size as an integer.
The detection study shows that with high contrast sections, such as silver impregnated trabecular bone, the 'flicker' method of setting the grey-level threshold gives an accurate binary representation of the trabecular bone. The magnification study shows that for the same range of box sizes ( $77 \mu \mathrm{m}$ to $2652 \mu \mathrm{m})$, initial resolution has no effect on the estimation of the fractal dimension, over the magnification range used in this study. The angle of presentation study shows that the architecture of trabecular bone is not sufficiently anisotropic, for the angle of presentation to the image analyser, to significantly affect the estimation of the fractal dimension. These studies mean that there does not have to be absolute control over how the sample is presented to the image analyser. However, as in all morphometric studies it is best if a uniform approach is taken to minimise bias and random error in the measurements.

Using 3 straight-line segments (D1, D2 and D3) to describe the data of the modified Richardson plot is statistically more accurate than one straight line. This is reflected in the values for the regression coefficient (R), which are greater for the sectional fractal dimensions than for a single fractal dimension. A previous study by Fazzalari and Parkinson (1997) showed that 2 'break points' correlate well with structural entities in the cancellous structure. The interpretation is that the 3 fractals (D1, D2 and D3) each describe a different compartment of the cancellous structure.

The data presented in this paper, to determine a stable and reproducible methodology for fractal analysis of trabecular bone, show that trabecular bone is effectively fractal over a defined range of scale. Also, within this range, there is more than 1 fractal dimension, describing spatial structural entities.

Unlike conventional histomorphometry, fractal analysis is a model independent method for describing a complex multifaceted structure using an objective methodology within clearly defined operational parameters. Although this study describes a method specifically for trabecular bone, it can be adapted to the fractal analysis of other biological systems. This may be at the cell, tissue or organ level, giving insight to complex processes. Conventional histomorphometric and stereological techniques would be complemented by fractal analysis.

A preliminary report of some of the data (Parkinson et al., 1999) has been presented at the $\mathrm{X}^{\text {th }}$ International Congress for Stereology, Melbourne, Australia, 1-4 November 1999. 


\section{REFERENCES}

Buczkowski S, Kyriacos S, Nekka F, Cartilier L (1998). The modified box-counting method: Analysis of some characteristic parameters. Pattern Recognition 31:411-8.

Chung HW, Chu CC, Underweiser M, Wehrli FW (1994). On the fractal nature of trabecular structure. Med Phys 21: 1535-40.

Fazzalari NL, Parkinson IH (1996). Fractal dimension and architecture of trabecular bone. J Pathol 178:100-5.

Fazzalari NL, Parkinson IH (1997). Fractal properties of subchondral cancellous bone in severe osteoarthritis of the hip. J Bone Miner Res 12:632-40.
Kaye BH (1987). Discovering Texture Fractals. In: BH Kaye, ed. A random walk through fractal dimensions. Ch. 2.3. Germany: VCH Publishers, 25-37.

Majumdar S, Weinstein RS, Prasad RR (1993). Application of fractal geometry techniques to the study of trabecular bone Med Phys 20:1611-9. [published erratum appears in Med Phys 1994 Mar; 21(3), 491].

Mandelbrot BB ed. (1977). The fractal geometry of nature. New York: W.H. Freeman.

Vicsek T (1998). Scaling in biology. Keynote address: Fractal 98 "Complexity and fractals in science" $5^{\text {th }}$ Interdisciplinary Conference, Malta.

Weinstein RS, Majumdar S (1994). Fractal geometry and vertebral compression fractures. J Bone Miner Res 9:1797-802. 